\title{
A Search for Trends in Cometary Dust Emission
}

\author{
C.M. Lisse $^{\mathrm{a}}$, M.F. A'Hearn ${ }^{\mathrm{b}}$, Y.R. Fernandez ${ }^{\mathrm{c}}$, S.B. Peschked \\ aSpace Telescope Science Institute, Servicing Mission Office, 3700 San Martin Drive, \\ Baltimore, MD 21218 lisse@stsci.edu \\ b University of Maryland, Department of Astronomy, College Park, MD 20742 ma@astro.umd.edu \\ c Institute for Astronomy, University of Hawaii , 2680 Woodlawn Drive, Honolulu, HI 96822 \\ yan@ifa.hawaii.
}

dISO Data Centre, Villafranca Del Castillo, ESA Satellite Tracking Station, P. O. Box (Apdo.) 50727, E-28080 Madrid, Spain speschke@iso.vilspa.esa.es

We present the results of searching for statistical trends in the properties of dust emitted by 9 comets as determined using mid-IR observations. Trends in the aggregate dataset of dust PSD, total emission rate, and emission rate vs time were found versus dynamical class, with the short period comets slowly emitting mainly large, dark dust particles while the LP comets emitted most of their dust surface area (but not mass) rapidly in small, high albedo particles. These differences may be due to the effects of cometary evolution on the structure of the cometary surface and/or the depletion of cometary volatiles, although caveats concerning our currently small (but growing) dataset and the role of potentially important selection effects in our results.. These results are consistent, however, with a larger sample of 46 infrsred comet observations found in the literature.

\section{Introduction}

Studies of the physical properties of cometary dust are important for understanding the phenomena of comets as a class, and for understanding the formation and evolution of comets and the solar system. The quantities we can derive from infrared observations of the dust include the dust composition, mass, particle size distribution, and emission history. Given a large enough statistical population of observations, we can use the results of the measurements to understand the role of comets in the present day solar system, including, e.g., the contribution of cometary dust to the interplanetary dust cloud, and the evolution and fate of highly aged comets. Since comets are among the most primitive bodies in the inner solar system, despite their evolution, we also want to use the results to understand the formation of the solar system from the proto-solar nebula, e.g., the total and relative abundance of rock forming material, the formation and evolution of the accreting icy planetesimals (comets), and chemical variations in the proto- solar nebula. We hope to learn more about the aging process of comets, e.g.: the effect of weathering and mantling on the cometary surface and the effect of collisions on the cometary population.

Combined thermal infrared and optical observations are sensitive to the $0.1-100$ um range of dust particle sizes [1],[2], [3]). The presence of a strong $8-13 \mu \mathrm{m}$ silicate emission feature is also an indicator of relatively small dust grains $(\leq 10 \mu \mathrm{m})$, as is a spectral color temperature elevated more than $10 \%$ above the local equilibrium temperature, or a negative deviation from greybody behavior at $30-100 \mu \mathrm{m}$ [4]. A total albedo for scattering, defined as the ratio of the scattered luminosity to the total luminosity observed at a given phase angle, is $\sim 6 \%$ for large cometary particles (e.g., comets Austin and Encke) and $>12 \%$ for small particles (e.g. comets Levy, Hyakutake, and Hale-Bopp). Extensions of the dust tail morphology along the projected orbital velocity direction (i.e., "trails") are due to large, heavy particle emission, while morphologies with only anti-solar tails are indicators of small particle emission [3]. Trends in long term light curves yield independent estimates of the particle size distribution and emission rate [1],[2]). 
There is growing evidence, from the Giotto encounter with the P/Halley nucleus [5], the IRAS observations of C/IRAS-Araki-Alcock 1983H1 [6], the COBE/DIRBE large angular scale observations of 4 comets [1], and the sub-mm observations of comet C/Hyakutake 1999 B2 and C/Hale-Bopp 1995 O1 [7],[8], that the majority (by mass) of grains emitted by comets are visibly dark and large, requiring the use of mid- to far-IR observations to probe the nature of cometary dust. This conclusion is very different from the current paradigm created mainly from large optical surveys of periodic comets [9],[10], which concludes that the majority of cometary dust mass is emitted by the optically bright comets.

\section{Observations}

Our study of the statistical properties of cometary dust emission began with the large-scale photometric imaging of 4 comets detected in the COBE/DIRBE all-sky survey of $1989-1990$. These comets were selected only by our ability to detect them against the background in the COBE all sky survey, down to $\mathrm{V} \sim$ [12]. As summarized [1], C/OLR and C/Austin were found to emit only large, dark dust grains not detected in the visible, while C/Levy was more Halley-like in its emission of many small dust grains. That the comet $73 \mathrm{P} / \mathrm{SW}-3$ was detected at all by COBE was quite surprising, as it was optically fainter than 4 other short period comets that were not detected; it was argued [1] that this was due to the enhanced release of large, dark particles due to the incipient breakup of the comet during its next perihelion passage.

The present paper adds 5 more comets to our database of comets well-characterized using multiwavelength photometry in the thermal infrared (Table I). Included are the extremely bright long-period comets of 1996 and 1997, C/Hyakutake 1996 B2 [11] and C/Hale-Bopp 1995 O1 [2], [12]; and the closeapproaching periodic comets P/IRAS 1996, P/Encke 1997 [3], and P/Tempel-Tuttle 1998. Not included are comets like P/Wild2 1997, C/Utsunomiya 1997 T1, and C/LINEAR 1998 U5, which were observed by our group but only detected in broad-band $\mathrm{N}$ [13]. The reader is referred to the individual observational papers listed for each comet for the details of the observations and the photometric calibration.

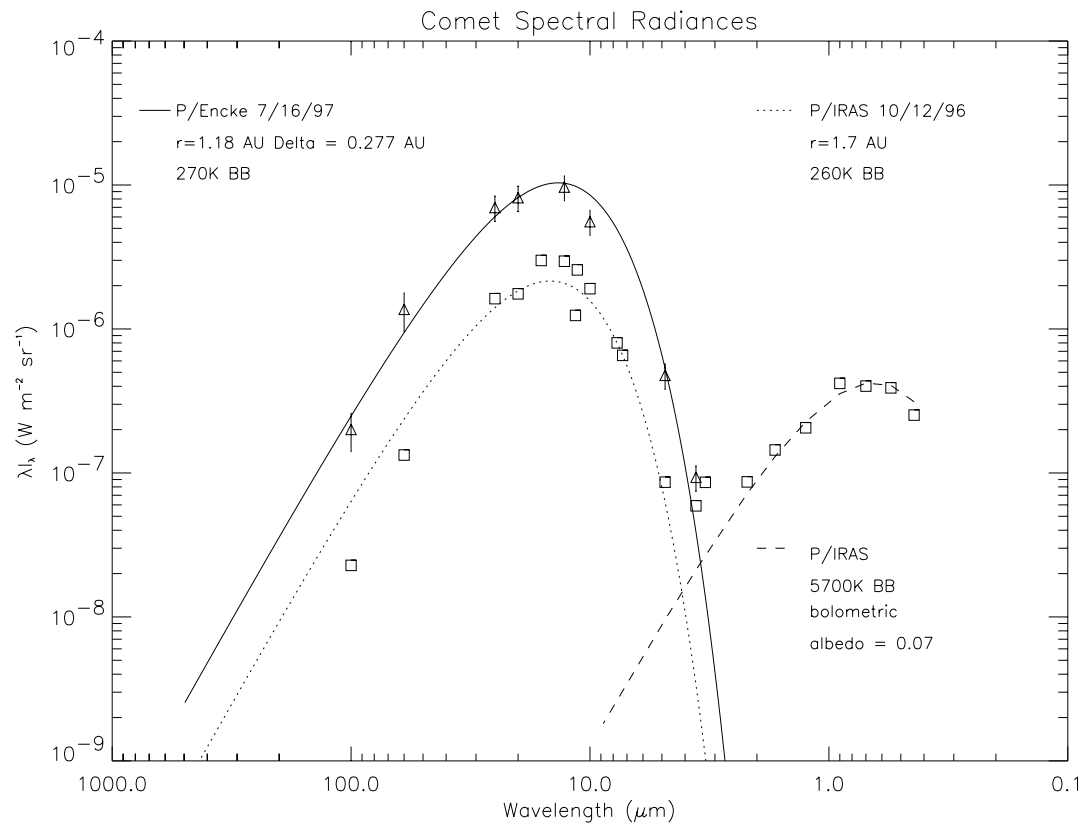

Figure 1 - Example of small and large particle dominated dust emission infrared spectral observations. a) Spectral energy distribution of comet P/IRAS 1996 as taken by ISO from $3-100 \mu \mathrm{m}$, at the NASA/IRTF 
from 1-3 $\mu \mathrm{m}$, and at the Lowell 42" on October 13, 1996 [3]. Squares - ISO observations with 20\% error bars. Dotted Curve - best-fit greybody to the infrared data, $20 \%$ hotter than the local equilibrium temperature, due to the presence of small, hot grains. From 8 to $13 \mu \mathrm{m}$, there is a strong emission feature due to silicates in small dust grains. At 60 and $100 \mu \mathrm{m}$, there is a strong fall off from the blackbody curve due to decreasing emission efficiency from small grains at large wavelengths. Dashed Curve - solar spectrum normalized to ISO observation at $1.25 \mu \mathrm{m}$. There is good agreement between the observed scattered light spectrum and a blackbody of $5700 \mathrm{~K}$, the average temperature of the Sun's emission. b) Spectral energy distribution of comet P/Encke 1997 as taken by ISO from $3-100 \mu \mathrm{m}$ on July 16, 1997, and at $5-20 \mu \mathrm{m}$ at the ESO $3.6 \mathrm{~m}$ on July 18, 1997 [3]. Triangles - ISO observations with $20 \%$ error bars. Solid curve - best fit greybody to the emission. The best fit greybody temperature is very close to the local equilibrium temperature, a strong indication that only large grains are being observed. There is no evidence of a silicate emission feature or long wavelength emissivity falloff.

Table I

Emitted Dust Properties for the 9 Comets In the IR Survey

\begin{tabular}{|c|c|c|c|c|c|c|c|c|}
\hline Comet & $\begin{array}{c}\text { Time } \\
\text { (UT Day) }\end{array}$ & $\begin{array}{c}\mathrm{r} \\
(\mathrm{AU}) \\
\end{array}$ & $\begin{array}{c}\mathrm{Tc} / \mathrm{Tb} \\
\mathrm{b}\end{array}$ & $\begin{array}{c}\text { Dust } \\
\text { Albedo } \\
(\%)\end{array}$ & $\begin{array}{c}\text { Silicate } \\
\text { Strength } \\
(\%)\end{array}$ & $\begin{array}{l}\text { QDust } \\
(\mathrm{kg} / \mathrm{sec})\end{array}$ & $\begin{array}{c}\text { Qgas } \\
(\mathrm{mol} / \mathrm{sec})\end{array}$ & Dust/Gas \\
\hline C/OLR (New) ${ }^{*}$ & 1989 Dec 20 & 1.0 & 1.06 & --- & $<10$ & 450 & $4 \times 10^{28}$ & 0.32 \\
\hline C/Austin (New) ${ }^{*}$ & 1990 May 15 & 0.94 & 1.08 & 6 & $\sim 10$ & 2400 & $1 \times 10^{29}$ & 0.79 \\
\hline $73 \mathrm{P} / \mathrm{SW}-3$ (SP) ${ }^{*}$ & 1990 Apr 15 & 1.07 & 1.05 & --- & $<20$ & $\sim 120$ & $<3 \times 10^{28}$ & $>0.12$ \\
\hline C/Levy (LP) $)^{\dagger}$ & 1990 Sep 20 & 1.12 & 1.24 & 12 & $>30$ & 6100 & $2 \times 10^{29}$ & 1.0 \\
\hline $\mathrm{C} /$ Hyakutake(LP) ${ }^{\dagger}$ & 1996 Mar 25 & 1.05 & 1.20 & 13 & $>50$ & 9000 & $2 \times 10^{29}$ & 1.7 \\
\hline 126P/IRAS $(\mathrm{H})^{\dagger}$ & 1996 Oct 13 & 1.71 & 1.25 & 16 & $>35$ & 300 & $3 \times 10^{27}$ & 3.3 \\
\hline C/Hale-Bopp(LP) $)^{\dagger}$ & 1996 Oct 31 & 2.54 & 1.30 & 39 & $>200$ & 140000 & $2 \times 10^{31}$ & 5.0 \\
\hline $2 \mathrm{P} /$ Encke (SP) ${ }^{*}$ & 1997 Jul 15 & 1.18 & 1.03 & 5 & $<10$ & 120 & $2 \times 10^{27}$ & 2.3 \\
\hline $\begin{array}{l}\text { 55P/Tempel-Tuttle } \\
\text { (SP) }\end{array}$ & 1998 Jan 21 & 1.15 & 1.03 & $5 ?$ & $<20$ & 210 & $3 \times 1027$ & 2.9 \\
\hline
\end{tabular}

* - Large particle dominated comet. $\dagger^{\dagger}$ - Small particle dominated comet. $\S-q>5$ AU comet.

N.B. : All Qdust, $\mathrm{D} / \mathrm{G}$ estimates are conservative, assuming minimum largest particle, with factor of $+100 \% /-50 \%$ errors, with the dominant systematic uncertainty in the dust outflow velocity.

\section{Models}

Results from the comet observations of the COBE mission [14],[1], have shown that simultaneous modeling of the shape and scattering/thermal emission spectrum of a comet's dust tail can constrain the silicate:carbonaceous composition ratio and particle size distribution of the dust. Our methods of spectral, dynamical, and temporal modeling of the thermal emission from the cometary dust have been extensively summarized in [1], where we demonstrate the ability to derive absolute dust production rates, dust-to-gas ratios, and rough compositional information for 4 comets using 1 - $300 \mu \mathrm{m}$ multiwavelength broad-band infrared photometric imaging obtained by the COBE/DIRBE instrument in 1989 - 1990. [2] demonstrates our ability to use the same modeling techniques for comet C/Hale-Bopp from ground based $1-20 \mu \mathrm{m}$ photometric measurements. 


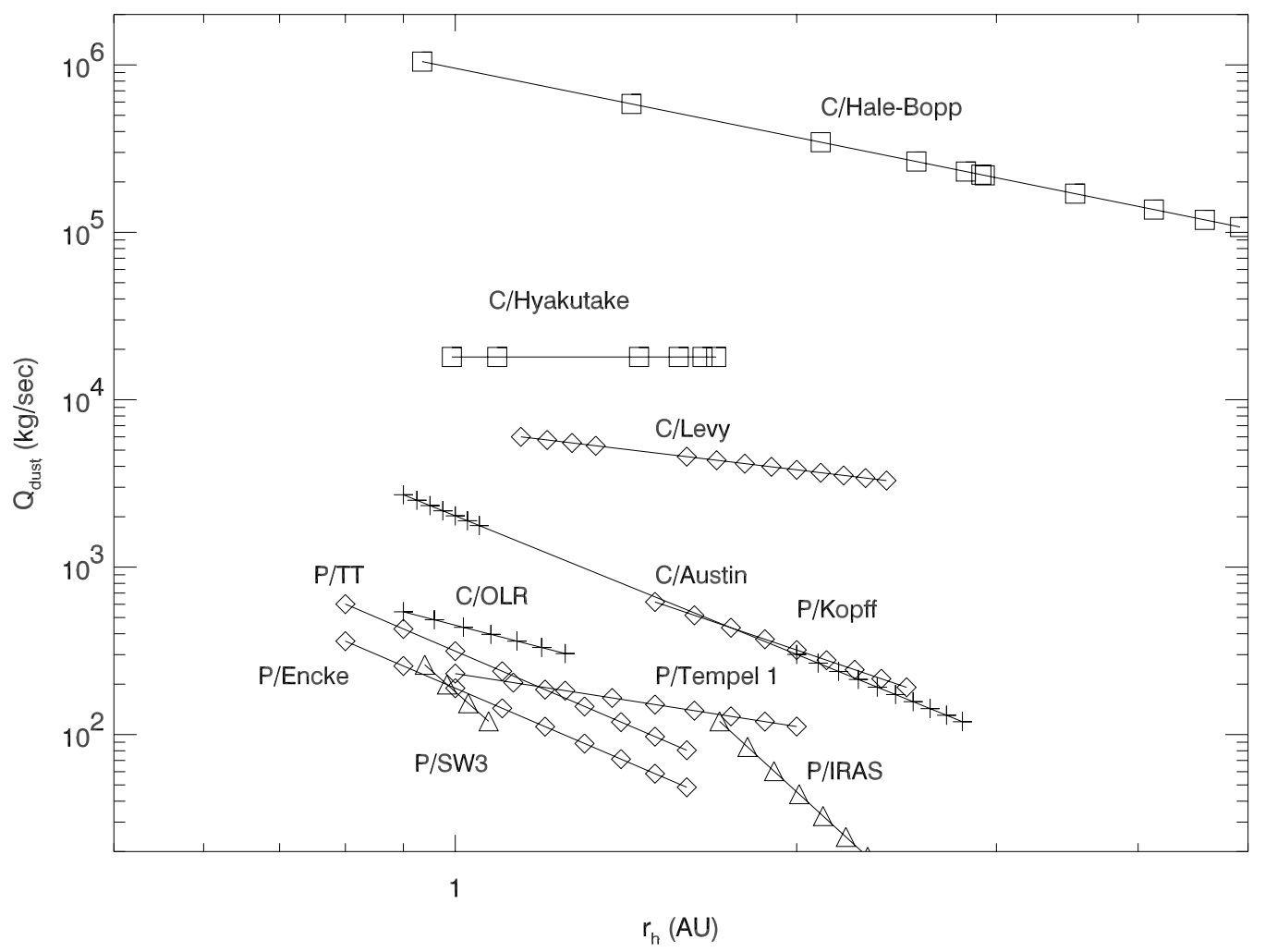

Figure 2 - Derived mid-infrared dust emission rate vs heliocentric distance for the 9 comets in our survey. There is a general trend of steeper slope and lower absolute emission rate for periodic comets versus new or long period comets. A possible selection effect that may affect the results is our ability to detect and observe fainter periodic comets due to their known positions and orbits - although the 4 comets from the COBE dataset were detected in an unbiased all-sky survey.

We present here the aggregate results of modeling our IR observations of 9 different comets (Table I, Figure 2). Our broad-band multi-wavelength photometry observations can be explained by dust with similar silicate:carbonaceous composition ratios of 2 to $3: 1$, but two very different dust particle size distributions (PSD's), the [15] and the $\mathrm{m}^{-0.63}$ (or $1 / \beta ;[1]$ ) distributions. While both these PSD's are mass dominated by the largest (radius $>100 \mathrm{um}$ ) particles, the emitted surface area (and optical brightness) is dominated by either $0.1-10$ um particles in "dusty comets" like 1P/Halley or $>10$ um particles in "gassy comets" like 2P/Encke. The morphology of the coma and tail for the dusty comets is typically "normal", i.e. dominated by a bright, linear tail pointing roughly in the anti-solar direction; this tail is absent or weak and in addition to a curved tail pointing along the direction of orbital motion in the "gassy" comets.

\section{$4 \quad$ Results and Discussion}

Of the five new well-measured comets in our infrared survey of cometary dust emission, the intermediate-aged long period comets C/Hyakutake 1996B2, P/IRAS 1996, and C/Hale-Bopp 1995O1 demonstrated high rates of dust emission dominated by small particles, including pronounced silicate features, long-wavelength emissivity falloffs, and large superheats, while the extremely processed short period comets $\mathrm{P} / \mathrm{Sch}$ wassmann-Wachmann 3, P/Encke, and $\mathrm{P} /$ Tempel-Tuttle emitted mainly large dust particles at relatively low rates. The dust from Comet Hale-Bopp was slightly anomalous in that the 
particles seemed especially dense and small [2], although of normal composition compared to other comets. Taken in aggregate, there appears to be a real difference in the particle size distributions for our 9 comet dataset of the dust emitted by the new and short period comets, which emit mainly large, dark dust particles, versus the small, bright dust emitted by the long period comets. The new comets also appear to have lower $\mathrm{D} / \mathrm{G}$ ratios than the others.

Comparison of our results to the two classes of comets found in the optical polarimetric survey of Levasseur-Regourd [16] shows an excellent match of our large particle dominated emission with their "gassy, low polarization comets" and our small particle dominated emission with their "dusty, high polarization comets". This is consistent with the expectation that small particles have a higher albedo and polarization per unit dust mass [4],[12]. Hale-Bopp stands out as having unusually high polarization and albedo, which is consistent with our models showing it had unusually solid, small dust [2]. The strength of the $8-13 \mu \mathrm{m}$ silicate emission feature versus the continuum is known to be inversely proportional to the dust particle size [17]; for the 5 of our comets also observed in the silicate feature at $8-13 \mu \mathrm{m}$ by Hanner et al. [18], we find good agreement between our assignment of large or small particle dominated emission and their finding of large or small amplitude of the silicate feature.

Concern that our results may be preliminary due to small sample size and selection effects led us to search the literature for other infrared comet observations. (Note that while the 5 new comets were selected on the basis of their expected brightness and known orbits, the original 4 comets of the database, detected in the COBE all sky survey of 1989 - 1990, were selected only by their IR brightness against the background.) In Table II we present the results of searching for thermal IR observations of comets in the literature (c.f. [19] for references), ordered by whether small or large particle emission was evident via the superheat/silicate feature strength/long wavelength emissivity falloff criteria, and ordered within these two groups by perihelion distance. For the more than 40 literature comets observed, we again find the same distinction in the emitted dust between the SP + New and the LP comets. The Halley family comets seem to be split in their behavior.

Table II

Cometary Dust Size Distributions Well Observed To Date in the Mid-IR

\begin{tabular}{|c|c|c|c|c|c|c|c|}
\hline Proper Name & Tperi & $\begin{array}{c}\mathrm{q} \\
(\mathrm{AU}) \\
\end{array}$ & $\mathrm{e}$ & $\begin{array}{c}\mathrm{P} \\
(\mathrm{yr})\end{array}$ & $\begin{array}{c}\mathrm{i} \\
(\mathrm{deg}) \\
\end{array}$ & Tisserand & $\begin{array}{c}\text { Dynamical } \\
\text { Type }\end{array}$ \\
\hline \multicolumn{8}{|l|}{$\frac{\text { Large Particle }}{\underline{\text { Dominated }}}$} \\
\hline 2P/Encke & 1994 Feb 9 & 0.33 & 0.85 & 3.28 & 11.9 & 3.03 & SP, old \\
\hline $\begin{array}{l}\text { 23P/Brorsen- } \\
\text { Metcalf }\end{array}$ & 1989 Sept 11 & 0.48 & 0.97 & 70.5 & 19.3 & 1.12 & $\mathrm{H}$ \\
\hline $\begin{array}{l}\text { 73P/Schwassmann } \\
\text {-Wachmann3 }\end{array}$ & 1995 Sept 22 & 0.93 & 0.69 & 5.34 & 11.4 & 2.78 & SP, old \\
\hline 103P/Hartley 2 & 1991 Sept 12 & 0.95 & 0.72 & 6.26 & 9.25 & 2.64 & SP, young \\
\hline 55P/Tempel-Tuttle & 1965 Apr 30 & 0.98 & 0.90 & 32.9 & 163. & 1.65 & $\mathrm{H}$ \\
\hline $\begin{array}{l}\text { 26P/Grigg- } \\
\text { Skjellerup }\end{array}$ & 1992 July 22 & 0.99 & 0.66 & 5.10 & 21.1 & 2.81 & $\mathrm{SP}$, mid \\
\hline $\begin{array}{l}107 \mathrm{P} / \text { Wilson- } \\
\text { Harrington }\end{array}$ & 1992 Aug 22 & 1.00 & 0.62 & 4.29 & 2.79 & 3.09 & SP \\
\hline $\begin{array}{l}\text { 21P/Giacobini- } \\
\text { Zinner }\end{array}$ & 1992 Apr 13 & 1.03 & 0.71 & 6.61 & 31.8 & 2.47 & $\mathrm{SP}$, mid \\
\hline 46P/Wirtanen & 1991 Sept.20 & 1.08 & 0.65 & 5.50 & 11.7 & 2.82 & SP, young \\
\hline 24P/Schaumasse & 1993 Mar 4 & 1.20 & 0.70 & 8.22 & 11.8 & 2.51 & SP, old \\
\hline 7P/Pons-Winnecke & 1989 Aug 20 & 1.26 & 0.63 & 6.38 & 22.3 & 2.69 & SP, Middle \\
\hline $\begin{array}{l}\text { P/1983 V1 } \\
\text { Hartley-IRAS }\end{array}$ & 1984 Jan 9 & 1.28 & 0.83 & 21.5 & 95.7 & 0.809 & SP \\
\hline $\begin{array}{l}67 \mathrm{P} / \text { Churyumov- } \\
\text { Gerasimenko }\end{array}$ & 1989 June 18 & 1.30 & 0.63 & 6.59 & 7.12 & 2.75 & SP,young \\
\hline
\end{tabular}




\begin{tabular}{|c|c|c|c|c|c|c|c|}
\hline 10P/Tempel 2 & 1994 Mar 16 & 1.48 & 0.52 & 5.48 & 12.0 & 2.97 & SP, old \\
\hline 9P/Tempel 1 & 1994 July 3 & 1.49 & 0.52 & 5.50 & 10.6 & 2.97 & SP, old \\
\hline 28P/Neujmin 1 & 1984 Oct 8 & 1.55 & 0.78 & 18.2 & 14.2 & 1.55 & $\mathrm{H}$ \\
\hline 81P/Wild 2 & 1990 Dec 16 & 1.58 & 0.54 & 6.37 & 3.24 & 2.88 & SP, young \\
\hline 22P/Kopff & 1996 July 2 & 1.58 & 0.54 & 6.45 & 4.72 & 2.87 & SP, old \\
\hline \multicolumn{7}{|l|}{ Harrington } & SP, mid \\
\hline C/1989 X1 Austin & 1990 Apr 10 & 0.35 & 1.000225 & & 59.0 & 0.375 & New \\
\hline $\begin{array}{l}\text { C/1975 N1 } \\
\text { Kobayashi-Berger- } \\
\text { Milon }\end{array}$ & 1975 Sept 5 & 0.43 & 1.000097 & & 80.8 & 0.128 & New \\
\hline $\begin{array}{l}\text { C/1974 C1 } \\
\text { Bradfield }\end{array}$ & 1974 Mar 18 & 0.50 & 0.999697 & & 61.3 & 0.425 & New \\
\hline C/1989 Q1 OLR & 1989 Nov 12 & 0.64 & 1.000020 & & 90.1 & 0.002 & New \\
\hline $\begin{array}{l}\text { C/1983 H1 IRAS- } \\
\text { Araki-Alcock }\end{array}$ & 1983 May 21 & 0.99 & 0.990115 & & 73.3 & 0.407 & LP \\
\hline $\begin{array}{l}\text { C/Wilson C/1986 } \\
\text { P1-A }\end{array}$ & 1987 Apr 21 & 1.20 & 1.000312 & & 147. & 1.14 & New \\
\hline C/1983 J2 IRAS & 1983 Jan 19 & 1.42 & 1.0 & & 152. & 9999 & New? \\
\hline $\begin{array}{l}\text { C/1980 Y2 } \\
\text { Panther }\end{array}$ & \multicolumn{7}{|c|}{ Panther } \\
\hline C/1983 O2 IRAS & 1983 Nov. 28 & 2.25 & 1.000196 & & 121. & 0.951 & New \\
\hline C/1980 E1 Bowell & 1982 Mar. 12 & 3.36 & 1.057322 & & 1.66 & 2.22 & New \\
\hline C/1983 O1 Cernis & 1983 July 21 & 3.32 & 1.001968 & & 135. & 1.59 & LP \\
\hline \multicolumn{8}{|c|}{ Total : 16 SP, 3 H, 8 N, 3 LP } \\
\hline \multicolumn{8}{|l|}{$\frac{\text { Small Particle }}{\text { Dominated }}$} \\
\hline 1P/Halley & 1986 Feb 9 & 0.59 & 0.97 & 76.0 & 162 & 1.19 & $\mathrm{H}$ \\
\hline $\begin{array}{l}\text { 126P/1983 M1 } \\
\text { IRAS }\end{array}$ & 1983 Aug 2 & 1.70 & 0.70 & 13.2 & 46.2 & 1.96 & $\mathrm{H}$ \\
\hline 19P/Borrelly & 1994 Nov 1 & 1.37 & 0.62 & 6.88 & 30.3 & 2.57 & SP, Young \\
\hline $\begin{array}{l}\text { 38P/Stephan- } \\
\text { Oterma }\end{array}$ & 1980 Dec 5 & 1.57 & 0.86 & 37.7 & 18.0 & 1.89 & $\mathrm{H}$ \\
\hline 4P/Faye & 1991 Nov 16 & 1.59 & 0.58 & 7.34 & 9.09 & 2.75 & SP, Young \\
\hline $\begin{array}{l}\text { D/1993 } \\
\text { Shoemaker-Levy } 9\end{array}$ & 1994 Mar 28 & 5.38 & 0.21 & 17.8 & 5.89 & 2.99 & $\mathrm{SP} \S$ \\
\hline $\begin{array}{l}\text { 29P/Schwassmann } \\
\text {-Wachmann } 1\end{array}$ & 1989 Oct 26 & 5.77 & 0.045 & 14.9 & 9.37 & 2. 99 & SP $\S$ \\
\hline P/Chiron & 1996 June 15 & 8.46 & & & & & SP $\S$ \\
\hline $\begin{array}{l}\text { C/1973 E1 } \\
\text { Kohoutek }\end{array}$ & 1973 Dec 28 & 0.14 & 1.000008 & & 14.3 & 0.453 & New \\
\hline $\begin{array}{l}\text { C/1975 V1-A } \\
\text { West }\end{array}$ & 1976 Feb 25 & 0.20 & 0.999971 & & 43.1 & 0.402 & LP \\
\hline $\begin{array}{l}\text { C/1996 B2 } \\
\text { Hyakutake }\end{array}$ & 1996 May 1 & 0.23 & 0.999758 & & 125. & 0.346 & LP \\
\hline $\begin{array}{l}\text { C/1969 Y1 } \\
\text { Bennett }\end{array}$ & 1970 Mar 20 & 0.54 & 0.996193 & & 90.0 & 0.038 & LP \\
\hline $\begin{array}{l}\text { C/1987 P1 } \\
\text { Bradfield }\end{array}$ & 1987 Nov 7 & 0.87 & 0.994736 & & 34.1 & 0.987 & LP \\
\hline $\begin{array}{l}\text { C/1995 O1 Hale- } \\
\text { Bopp }\end{array}$ & 1997 Apr 1 & 0.91 & 0.995069 & & 89.4 & 0.040 & LP \\
\hline
\end{tabular}




\begin{tabular}{lllllll} 
C/1990 K1 Levy & 1990 Oct 25 & 0.94 & 1.000417 & 132. & 0.795 & LP \\
C/1993 A1 & 1994 Jan 13 & 1.94 & 1.001779 & 125. & 0.982 & LP \\
Mueller & & & & & & \\
C/1983 N1 IRAS & 1983 May 3 & 2.42 & 0.999420 & 139. & 1.45 & LP \\
\hline
\end{tabular}

\section{Total : 4 SP, $3 \mathrm{H}, 1 \mathrm{~N}, 8 \mathrm{LP}$}

Well Observed $=$ Photometry From at least $>5-20 \mu \mathrm{m}$, or 8-13 $\mu \mathrm{m}$ Using Spectroscopy

Large Particle Dominated $=$ Silicate Emission/Continuum $<10 \%$ and $/$ or $\mathrm{T} / \mathrm{T}_{\mathrm{bb}}<1.10$

Small Particle Dominated $=$ Silicate Emission/Continuum $>10 \%$ and $/$ or $\mathrm{T} / \mathrm{T}_{\mathrm{bb}}>1.10$

$\mathrm{SP}=$ Short period comet; $\mathrm{LP}=$ Long period comet $\mathrm{H}=$ Halley Family, New = Dynamically New Comet

$\mathrm{P}=$ Photometric Observation, $\mathrm{S}=$ Spectroscopic Observation, $\mathrm{I}=$ Imaging Observation, $\S=\mathrm{q}>5 \mathrm{AU}$

Dynamical type - From [18]

There is no obvious trend in $\mathrm{i}, \mathrm{P}$, or e in Table 2 . The only obvious trend in $\mathrm{q}$ is that all comets with $\mathrm{q}$ $>5$ AU have small particle dominated emission. Using estimated nuclear radii from the literature ([20], [13]), we see no obvious trend with $\mathrm{R}_{\mathrm{N}}$. E.g., LP comet Hale-Bopp, SP comets Chiron and SW-1 are all thought to be large, with varying levels of activity, while LP comets Levy and Hyakutake were as small as the average SP comet like Encke or Tempel-Tuttle. Thus any variability of the emitted dust due to nuclear structure differences dependent on size - like degree of melting and internal differentiation, and degree of heterogeneity - appear to be small.
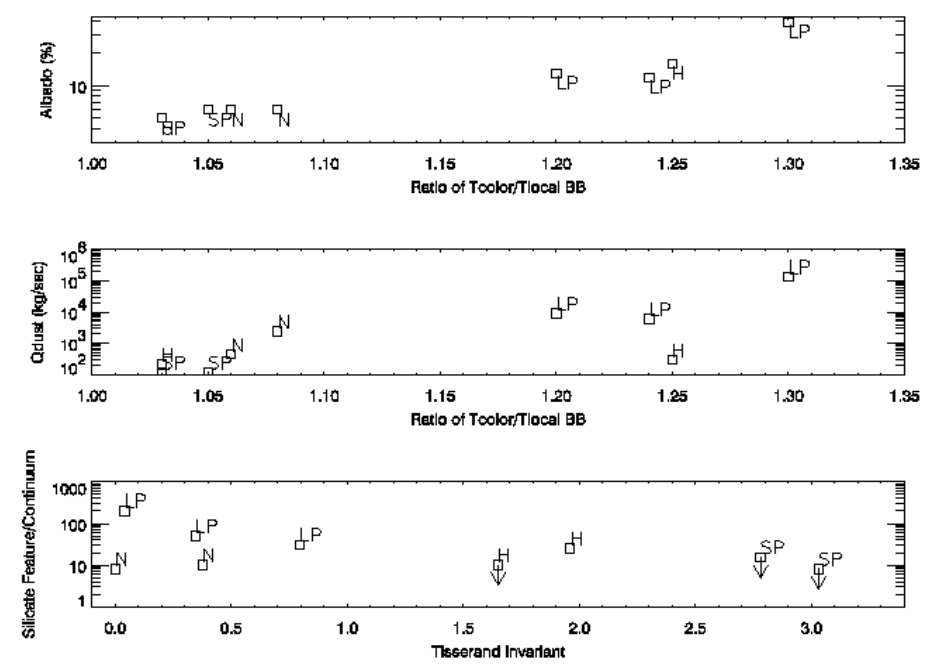

Fig 3 - A search for correlations in the observations of emtted cometary dust by dust albedo, silicate feature ampltude/continuum ratio, dust color temperature, Qdust, and Tisserand invariant of the parent comet. a) Comparison of the dust temperature and albedo (not shown is a correlation of the dust albedo and silicate feature strength, which demonstrates a similarly strong correlation); b) of the dust production rate and the temperature of the dust; and c) of the strength of the silicate feature and the Tisserand. There are clear trends and distinct groups by dynamical class - the LP comets produce the most dust with the hottest color temperature, highest albedo, and largest silicate feature. The New and Short Period comets produce little dust and what they do produce is cold, low albedo, and without much silicate feature. The Halley comets are between the two extremes.

The results of our literature search suggest that comparison of dust comae color temperature, silicate feature, superheat, and emissivity falloff at long wavelengths to cometary age and orbit for the 9 comets in our survey may yield some clues as to the formation and evolution of comets in the solar system (Figure 
3). We do indeed find strong correlations between the temperature, albedo, and silicate strength of the emitted dust (brighter dust is hotter and has a larger silicate feature), between the dust production rate and the temperature of the dust (hotter dust is produced more rapidly), and the strength of the silicate feature and the Tisserand invariant (largest silicate feature and smallest dust particles are emitted by the LP comets) for the 9 comets in our survey .

What, then, leads to the very different dust emitted by the long-period group of comets? One possible answer is that the presence of small grains in a comet may be an indicator of the evolutionary state of a comet, with smaller grains originating mainly from comets with highly evolved mantles and jet activity on their surface created after numerous perihelion passages. (Whether the small particles are produced in the violent emission associated with jets or are due to chemical differences in the jets is not clear, however.) After many passages, though, the reservoir of volatile material runs out, and the jets turn off with age.

The similarity in the dust albedo for 73P/SW-3, 2P/Encke [3], 55P/Tempel-Tuttle, and that found for the $1 \mathrm{P} /$ Halley nuclear surface $\left(\mathrm{p}_{\mathrm{V}} \sim 5 \% ;[5]\right)$, coupled with the preponderance of very large particles in the material emitted by the short period comets, suggest that the dust emitted is little modified from the that on the nuclear surface. I.e., large chunks of the comet's surface are being emitted slowly and relatively unchanged into the coma for the short epriod comets. In the other extreme of the dusty comets, we expect violent jet activity and/or the presence of ephemeral icy volatiles in the dust lead to changes in the emitted dust from that on the nuclear surface. Large increases in polarization and albedo in cometary jets, suggesting the production of excess small particles, has been found in the highly active comets Hale-Bopp [21] and Halley [22].

The evolutionary model also explains the increasing slope of the dust emission rate dependence with heliocentric distance for the more evolved short period comets (Figure 2), as the surface temperature has to be increased to higher values to drive off the denser mantle and/or scarcer volatiles in these comets. On the other hand, the large q short period comets, with surface temperatures unable to drive sublimation of the majority volatile $\mathrm{H}_{2} \mathrm{O}$, age very slowly and are locked into a state similar to the long-period comets.

A test of this paradigm can be found in the transition-case Halley family comets like P/IRAS, which seems to emit small dust typical of the long period comets. Since this comet has a relatively short period of 13.2 years, which will, if anything, most likely decrease along with the semi-major axis of the orbit as it becomes increasingly locked into the 2:1 resonance of the typical Jupiter family comet. Thus future monitoring of the dust emission from P/IRAS should show a change from small- to large-particle dominated emission as the comet ages. Another useful test will be an extended 1- $300 \mu \mathrm{m}$ photometric imaging and $8-13 \mu \mathrm{m}$ spectroscopic survey of a large number ( $>50)$ of periodic and new comets using a single dedicated, well calibrated instrument, allowing us to improve the statistics and reduce the systematics of our search, such as will be possible with the upcoming SIRTF mission.

\section{Conclusions}

Our infrared observations of dust emission from 9 comets can be explained by particles with similar composition ratios but two very different PSD's. While both these PSD's are mass dominated by the largest (radius $>100 \mathrm{um}$ ) particles, the emitted surface area (and optical brightness) is dominated by either 0.1 10 um particles in "dusty comets" or > 10 um particles in "gassy comets". There is good agreement between the results of our small/large particle dominated classification and the high/low polarization comets of Levasseur-Regourd et al. [16] and the strong/weak silicate feature comets reported by Hanner et al. [18] and Williams et al. [12]. Trends in the aggregate dataset of dust PSD, total emission rate, and emission rate vs time were found versus dynamical class, with the short period comets slowly emitting mainly large, dark dust particles while the LP comets emitted most of their dust surface area (but not mass) rapidly in small, high albedo particles. These differences may be due to the effects of cometary evolution on the structure of the cometary surface and/or the depletion of cometary volatiles, although caveats concerning our currently small (but growing) dataset and the role of potentially important selection effects 
in our results. To partially address these caveats, we have found 46 infrared studies of cometary dust emission in the literature, which, when classified by cometary class, showed evidence of small or large particle dominated dust emission consistent with the trends found in our database.

\section{Acknowledgments}

This work was supported by NASA Grant NAG-53361 for ISO data analysis and by an ESO guest scientist grant in March 1997.

\section{References}

[1] Lisse, C.M., M.F. A'Hearn, M.G. Hauser, T. Kelsall, D.J. Lien, S.H. Moseley, W.T. Reach, R.F. Silverberg 1998. Infrared Observations of Comets by COBE. Astrophys. J 496, 971 -991.

[2] Lisse, C.M., Y. R. Fernandez, M. F. A'Hearn, T. Kostiuk, T. A. Livengood, H. U. Kaufl, W.F. Hoffmann, A. Dayal, M. E. Ressler, M. S. Hanner, G.G. Fazio, J.L. Hora, S. Peschke, E. Grun, L.K. Deutsch, 1999. Infrared Observations of the Dust Emitted By Comet Hale-Bopp. Earth, Moon, and Planets 78:1-3, 251 - 257

[3] Lisse, C.M., M.F. A'Hearn, Y.R. Fernandez, E. Gruen, H.U. Kaufl, T.Kostiuk, D.J. Lien, D.J. Osip, S.B. Peschke, R.G. Walker, "ISO Measurements of Dust Emitted From Periodic Comets 126P/IRAS (1996) and 2P/Encke (1997)", Icarus (in press)

[4] Mason, C.G., Gehrz, R.D., Ney, E.P., Williams, D.M., and Woodward, C.E. 1998. The temporal development of the pre-perihelion infrared spectral energy distribution of comet Hyakutake (C/1996 B2). Astrophys. J 507, 398 - 403

[5] Keller, H.U., W.A. Delamere, M.J. Reitsema, W.F. Huebner, H.U. Schmidt 1987. Comet P/Halley's Nucleus and Its Activity. Astron. Astrophys 187, 807 - 823.

[6] Walker, R.G., H.H. Aumann, J. Davies, S. Green, T. DeJung, J.R. Houck, B.T. Soifer 1984. Observations of comet IRAS-Araki-Alcock 1983d. Astrophys. J 278, L11 - 14.

[7] Jewitt, D.C., and Matthews, H.E., 1997. Submillimeter Continuum Observations of Comet Hyakutake (1996B2). Astron. J 113, 1145 - 1151

[8] Jewitt, D.C., and Matthews, H.E., 1997. Particulate Mass Loss From Comet HaleAstron. J 117, 1056 - 1062.

[9] A'Hearn, M.F., R.L. Millis, D.G. Schleicher, D.J. Osip, and P.V. Birch 1995. The Ensemble Properties of Comets: Results from Narrowband Photometry of 85 Comets, 1976-1992. Icarus 118, 223 - 270.

[10] Fink, U, and Hicks, M.D., 1996. A survey of 39 comets using CCD spectroscopy. Astrophys. J., 459, 729-743

[11] Lisse, C. M., Y. R. Fernández, A. Kundu, M. F. A'Hearn, A. Dayal, L.K. Deutsch, G.G. Fazio, W.F. Hoffmann, J.L. Hora 1999. The Nucleus of Comet Hyakutake (C/1996 B2). Icarus 140, 189 - 204

[12] Williams, D.M., C.G. Mason, R.D. Gehrz, T.J. Jones, C.E. Woodward, D.E. Harker, M.S. Hanner, D.H. Wooden, F.C. Witterborn, H. M. Butner 1997. Measurement of Sub-micron Grains in the Coma of Comet Hale-Bopp C/1995 O1 During 1997 February 15-20 UT. Astrophys. J 489, L91 - 94.

[13] Fernandez, Y.R., Physical Properties of the Nuclei of Comets, PhD Thesis, 1999

[14] Lisse, C.M., H.T. Freudenreich, M.G. Hauser, T. Kelsall, S.H. Moseley, W.T.Reach, R.F. Silverberg 1994. Infrared Observations of Comet Austin (1990V) by the COBE/Diffuse Infrared Background Experiment. Astrophys. J 432, L71 - 74

[15] McDonnell, J..A.M., P.L. Lamy, , and G.S. Pankiewicz 1991. Physical Properties of Cometary Dust. In Comets in the Post-Halley Era (Bamberg Meeting 
Proceedings), (R.L. Newburn, M. Neugebauer, and J. Rahe, Eds.), pp. 1043 1073. Kluwer Academic, Dordecht.

[16] Levasseur-Regourd, A.C., E. Hadamcik, J.B. Renard 1996. Evidence for Two Classes of Comets From Their Polarimetric Properties at Large Phase Angles. Astron. Astrophys 313, 327 - 333.

[17] Gilman, R.C. 1974. Planck mean cross-sections for four grain materials. Astrophys. J 268, 397 - 403.

[18] Hanner, M.S., D.K. Lynch, and R.W. Russell 1994. The 8 - $13 \mu \mathrm{m}$ Spectra of Comets and the Composition of Silicate Grains. Astrophys. J 425, 274 - 285.

[19] Lisse, C.M., Infrared Observations of Cometary Dust by COBE, Phd Thesis, 1992

[20] Levison, H.F. and Duncan, M.J. 1994. The long-term dynamical behavior of short-period comets. In Origins of Solar Systems Workshop: The Origin, Evolution, and Detectability of Short period Comets, (SEE N94-11628 01-90), 1-36.

[21] Meech, K.J., Physical Propertieds of Cometary Nuclei, ACM 1996 Proceedings (in press)

[22] Jones, T.J., and Gehrz, R.D., 2000. Infrared Imaging Polarimetry of Comet C/1995 01 (Hale-Bopp)Icarus 143, 338 - 346.

[23] Levasseur-Regourd, A.C., E. Hadamcik, J.B. Renard 1999Similarities Between in situ measurements of locsl dust light scattering and dust flux impact data within the coma 1P/Halley. Astron. Astrophys 348, 636 - 641. 